\title{
Cómo configura una mediana empresa su estrategia ambiental: Un estudio de caso en dos empresas de Bogotá, Colombia
}

FECHA DE RECEPCIÓN: 10 de enero FECHA DE APROBACIÓN: 2 de febrero Pp. 56-73

Luisa Fernanda Latorre Carrillo* Andrés Camilo Pulido Hernández** Carlos Eduardo Moreno Mantilla***

How does a medium-sized enterprise shape an envirunmental strategy: a case study in two companies from Bagotá, Lalombia Lolombia

Mise en place de stratégies envirunnementales pour PME (Petites et Mayennes Entreprises) : étude de cas de deux entreprises de Bagotá, Calambie.

Camo uma empresa de porte médio configura sua estratégia ambiental: um estudo de caso em duas empresas de Bagotá, Colômbia

"Ingeniera Industrial, Universidad Nacional de Colombia

**Ingeniero Industrial, Universidad Nacional de Colombia.

${ }^{* * *}$ PhD., profesor asociado Departamento de Ingeniería de Sistemas e Industrial, Universidad Nacional de Colombia (Sede Bogotá). 


\section{RESUMEN}

El estudio toma como marco de referencia las teorías de Mintzberg (estrategias deliberadas y emergentes) y Strategy as Practice. Estas teorías son contrastadas frente a los hallazgos obtenidos en un estudio de caso múltiple de tipo descriptivo, realizado en dos PyMEs participantes del Programa de Gestión Ambiental Empresarial (PGAE) de la Secretaria Distrital de Ambiente de Bogotá. El estudio de caso comprendió una empresa de servicios y una de producción, apoyándose en la observación participativa y la entrevista semiestructurada como métodos de recolección de datos.

Los autores acompañaron a las empresas en el proceso de construcción de un plan estratégico ambiental, recopilando información que permitió determinar que las estrategias así construidas son deliberadas, lideradas por la gerencia media y que surgen como respuesta a las presiones ejercidas por los Stakeholders más influyentes para la organización. Los resultados obtenidos ofrecen evidencia sobre la aplicabilidad de estos marcos teóricos en el contexto colombiano y representan igualmente un aporte al estudio de la estrategia ambiental en este entorno.

\section{ABSTRACT}

This research study is based on Mintzberg's theories (emerging and deliberate strategies) as well as on Strategy as Practice. These theories are contrasted with the study outcomes of a multiple descriptive case done in two PyMES which are part of an Environmental Management Program of the Local Environmental Office of Bogota. This Case Study involved a service company and a production company which applied participative observation and a semi-structure interview as methods used to collect data.

The authors accompanied both companies in the process of designing a strategic environmental plan, collecting information which revealed that the applied strategies were deliberate, led by middle manage-ment and arose as an answer to pressures exerted by the most stakeholders for the company. The results of this study showed the applicability of these conceptual frameworks to Colombian contexts and represented an important support to the study of environmental strategies in such contexts.

\section{Palabras clave}

Estrategia ambiental Estrategia deliberada Estrategia emergente Strategy as practice PyMEs
Environmental strategy Deliberate strategy Emerging strategy Strategy as Practice PyMES 


\section{RESUMÉÉ}

Cette étude prend comme cadre de référence les théories de Mintzberg (stratégie délibérée et émergente) et de Strategy as Practice. Ces théories offrent des conclusions contrastées d'une étude de cas de type descriptif réalisé dans deux PME participant au Programme de Gestion Environnementale du Secrétariat à l'Environnement de la ville de Bogotá. L'étude de cas analyse deux entreprises, l'une dédiée aux services l'autre à la production, en utilisant une méthode d'observation participative et un entretien semidirigé pour la collecte des données.

Les auteurs de cette étude accompagnent en outre ces entreprises lors du processus de mise en place du Programme Stratégique Environnementale, participent à la collecte d'informations permettant de déterminer les stratégies à mettre en cuvre, et guident le personnel de cadres intermédiaires vers les résultats attendus par les parties prenantes les plus influentes de l'organisation. Ces résultats offrent la preuve de l'applicabilité de ces deux cadres théoriques dans le contexte colombien et représentent par ailleurs une contribution à l'étude de la stratégie environnementale dans ce milieu spécifique.

\section{RESUMO}

O estudo toma como marco de referência as teorias de Mintzberg (estratégias deliberadas e emergentes) e Strategy as Practice. Essas teorias são contrastadas em relação aos resultados obtidos em um estudo de caso múltiple do tipo descritivo realizado em duas empresas de pe-queno e médio porte participantes do Programa de Gestão Ambiental Empresarial da Secretaria Distrital Ambiental de Bogotá, através de. O estudo de caso compreendeu uma empresa de ser-viços e uma de produção, apoiando-se na observação participativa e a entrevista semiestrutura-da como métodos de coleta de dados.

Os autores acompanharam as empresas no processo de construção de um plano estratégico ambiental, coletando informação que permitiu determinar que as estratégias assim construídas são deliberadas, lideradas pela gerência média e que surgem como resposta as pressões exerci-das pelos acionistas mais influentes para a organização. Os resultados obtidos oferecem evi-dencia sobre a aplicabilidade destes marcos teóricos no contexto colombiano e representam igualmente um aporte ao estudo da estratégia ambiental neste ambiente.

\section{Mots clefs}

Stratégie environnementale Stratégie délibérée Stratégie émergente Strategy as Practice PME

\section{Palavras-chave}

Estratégia ambiental Estratégia deliberada Estratégia emergente Strategy as practice PyMEs 


\section{INTRODUCCIÓN}

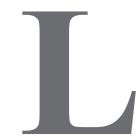
a configuración de una estrategia ambiental debe entenderse como el resultado de las interacciones dentro de una estructura intangible de personas y actividades, en donde se establecen acciones para alcanzar los objetivos ambientales de la organización. Las prácticas se relacionan con individuos o grupos que interactúan entre sí, dando forma a lo que se conoce como estrategia. La literatura clásica sobre estrategia ofrece en principio

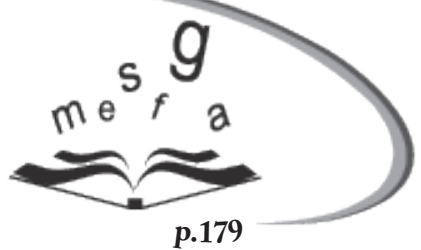

p.179 dos posturas: la teoría desarrollada por Michael Porter, con un enfoque en la estrategia deliberada, y la teoría planteada por Mintzberg, con un énfasis mayor que la anterior en la estrategia emergente. Recientemente, estas teorías han sido complementadas por el enfoque de Strategy as Practice, liderado por Jarzabkowski. Mientras las dos primeras se centran en entender si la construcción de una estrategia es producto de la planeación-estrategias deliberadas-, o si no lo es -estrategias emergentes-, la tercera tiene como fundamento observar la estrategia como una actividad, enfocándose en el hacer y en los individuos que la realizan.

Tomando como marco de referencia teórico la segunda ${ }^{1}$ y la tercera de estas corrientes, el presente estudio busca comprender cómo se estructuran las estrategias ambientales, contrastando la teoría frente a la evidencia empírica obtenida mediante un estudio de caso múltiple (Yin, 2003), apoyado en la técnica de observación participativa desarrollado en dos medianas empresas de la ciudad de Bogotá, una manufacturera y otra de servicios.

Las empresas estudiadas participan en el Programa de Gestión Ambiental Empresarial (PGAE), de la Secretaria Distrital de Ambiente (SDA) de la ciudad de Bogotá. Este es un programa voluntario en el que las empresas se vinculan para "mejorar su desempeño ambiental [...] yendo más allá del cumplimiento normativo, desarrollando actividades de carácter preventivo e incorporando la variable ambiental dentro de toda la estructura organizacional de la empresa" (Secretaria Distrital de Ambiente, s.f.).

En el primer nivel de este programa, las empresas emplearon herramientas básicas de diagnóstico que les permitieron identificar los impactos ambientales, así como capacitarse en normatividad ambiental y en los temas básicos de producción sostenible. Para el momento en que se inicia el estudio, en el mes de abril de 2014, las dos empresas estaban comenzando el segundo nivel del PGAE, en el que se busca que las empresas desarrollen estrategias ambientales para orientar el cambio de patrones de operación del negocio hacia la producción sostenible (Secretaria Distrital de Ambiente, s.f.).

Paralelamente al desarrollo del nivel II del PGAE, los autores acompañaron a las empresas en la formulación de un plan estratégico ambiental, lo cual los permitió descubrir las dinámicas internas de las organizaciones en el proceso de formulación estratégica ambiental. La importancia de estudiar la en este contexto, radica en que la literatura sobre este tema aplicada a organizaciones colombianas, es escasa, aún más en el caso de las PyMEs lo anterior contrastar en este contexto particular, las teorías planteadas fundamentalmente a partir del estudio de grandes organizaciones en países con economías desarrolladas (Moreno-Mantilla y Reyes, 2010).

Para responder al objetivo del estudio: ¿Cómo se configura un estrategia ambiental? Es necesario identificar los actores que participan en la configuración

\footnotetext{
${ }^{1}$ Los autores optan por esta delimitación del marco teórico, dado que Mintzberg incluye tanto estrategias deliberadas como emergentes ofreciendo un enfoque más amplio.
} 
de la misma, sus interacciones, las herramientas y acciones usadas y los tipos de estrategias configuradas, siendo las unidades de análisis cada una de las empresas estudiadas. Se esperaría encontrar que la configuración de la estrategia ambiental sea liderada por la alta gerencia (de Bakker \& Nijhof, 2002; Helfat et al., 2007), quien trabajará en conjunto con toda la organización; asi mismo, que la interacción entre los participantes se ajuste a las prácticas definidas por el marco de Strategy as Practice. Como consecuencia de la existencia clara de intenciones para la mejora del desempeño ambiental, las estrategias encontradas podrían verse como las que se definieron como estrategias deliberadas. Sin embargo, no se descarta la posibilidad de encontrar estrategias emergentes.

\section{FUNDAMENTOS TEÓRICOS}

$\mathrm{E}$ n 1996, Porter definió la estrategia como la creación de una posición única a través de la realización de diferentes actividades. La estrategia, es entonces un proceso en el que se establecen metas de largo plazo y planes de acción, que buscan mejorar el desempeño de la organización en el mercado. Todas las organizaciones cuentan con una estrategia y esta hace parte del proceso de toma de decisiones

p. 179

(Fuller-Love \& Cooper, 2000).

\subsection{Estrategia deliberada y estrategia emergente}

En 1985, Mintzberg y Waters (citado por Çetinkay y Kalkan, 2013), definieron cinco tipos de estrategias: emergentes, previstas, deliberadas, realizadas y no realizadas (Figura 1). Al establecer relaciones entre los planes de acción de una organización y las acciones independientes ejecutadas por la misma, se clasifica la estrategia como estrategia prevista y/o realizada. De este concepto se desprende la estrategia deliberada, la cual es una estrategia planeada -estrategia previstaque puede ser ejecutada -estrategia realizada-, o no ejecutada -estrategia no realizada-; y la estrategia emergente, que se define porque sus acciones son efectuadas -estrategia realizada-, aún sin la presencia de intenciones (Mintzberg y Waters, 1985). Esta última, muestra la flexibilidad y habilidad de la administración para reaccionar a circunstancias inesperadas (Fuller-Love \& Cooper, 2000).
Figura 1. Tipos de estrategias y sus relaciones.

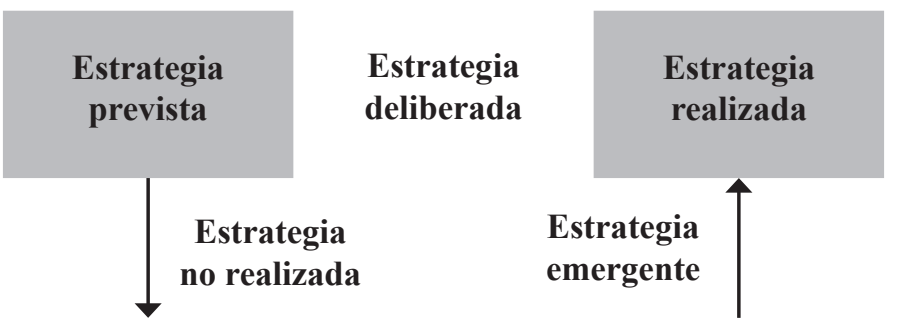

Fuente. Adaptado de Mintzberg y Waters (1985).

Para que una estrategia sea considerada puramente deliberada es decir, sus intenciones han sido cuidadosamente articuladas por la organización, se debe cumplir al menos con las siguientes tres condiciones: las intenciones deben estar definidas de forma precisa; deben ser colectivas; y el ambiente externo debe estar bajo control (Mintzberg \& Waters, 1985).

Para que una estrategia sea considerada puramente emergente quiere decir formada en ausencia de intenciones, sus acciones deben ser consistentes en el largo plazo, indicando, de esta manera, la existencia de estrategia (Mintzberg y Waters, 1985). Sin embargo, encontrar estrategias que cumplan con estas condiciones es difícil, por lo que en el presente trabajo se utilizará dicha terminología para categorizar estrategias que se acerquen a las definiciones.

A partir de las estrategias deliberadas y emergentes, Mintzberg y Waters (1985) proponen ocho tipos: 
- Estrategia planeada (deliberada):

En esta, los líderes centrales articulan sus intenciones de manera detallada y se encargan de difundir el contenido para su implementación.

\section{- Estrategia emprendedora (deliberada o emergente):}

Un actor impone su visión en la organización mediante el control y definición de las acciones a realizar.

\section{- Estrategia ideológica (deliberada):}

La visión es compartida colectivamente y todos los actores de la organización se identifican con esta.

\section{- Estrategia sombrilla (deliberada-emergente):}

Está presente en organizaciones donde los líderes no pueden imponer su visión, pero si acciones y límites para los actores.

\section{- Estrategia proceso (deliberada-emergente):}

El líder define quién y en qué contexto se hace la estrategia; esta es definida por los actores.

\section{- Estrategia desconectada (deliberada o emergente):}

En esta, cada actor de la organización establece sus acciones para realizar la estrategia.

\section{- Estrategia consenso (deliberada):}

Los actores convergen en el mismo tema.

\section{- Estrategias impuestas (deliberada):}

Un actor externo ejerce influencia en la organización determinando un patrón de acciones a seguir.

\subsection{Strategy as Practice}

La inconformidad frente a la teoría clásica, dio origen a la corriente teórica denominada Strategy as Practice (SAP) (Carter, Clegg, \& Kornberger, 2008). En SAP el enfoque se centra en los individuos, precisando que son estos los que al interior de los procesos organizacionales realizan la estrategia (Whittington, 2003). De esta manera, deja de ser solo un atributo más de la orga-nización y se concibe como una actividad ejecutada por las personas (Carter, Clegg, \& Kornberger, 2008).

Jarzabkowski y Spee (2009), explican que SAP "como área de investigación, se interesa con el hacer de la estrategia; quiénes la hacen, qué hacen, cómo lo hacen, qué utilizan y qué implicaciones tiene esto para definir la estrategia" [Traducción de los autores]. De esta manera, SAP define tres parámetros: practicantes (practitioners), práctica (practice), y prácticas (practices) (Jarzabkowski \& Spee, 2009).

Los practicantes son individuos tanto internos como externos de la organización, los cuales interactúan con circunstancias sociales relacionadas al hacer estrategia (Jarzabkowski, 2005). Estos no cuentan con un perfil específico de educación (Whittington, 2003), aunque es necesaria la existencia de competencias y conocimientos en los mismos. De esta manera, SAP se enfoca "en cómo los practicantes actúan, qué trabajo hacen, con quién interactúan y qué racionamiento práctico aplican" (Chía, 2004; Ezzammel \& Willmott, 2004; citados por Jarzabkowski, 2005). Debido a que la participación de los practicantes no es la misma, se establecerán como activos, siendo estos los actores líderes en la configuración de estrategia; y pasivos, los que acompañan a los líderes en el proceso.

La práctica será referida en el estudio como praxis con el fin de evitar confusiones con el término prácticas. Reckwitz (2002) y Sztompa (1991) (citado por Jarzabkowski y Spee, 2009), definen praxis como una actividad que interconecta micro-acciones de individuos y grupos con la organización en la cual las acciones contribuyen y se ubican. Dado que las praxis no están claramente definidas en SAP, los autores las conciben como aquellas actividades que influyen directamente 
en la estrategia de la organización, y son recurrentes y habituales para esta (Jarzabkowski \& Spee, 2009).

Finalmente, Jarzabkowski (2005) hace referencia a las prácticas como herramientas e instrumentos usados para hacer estrategia, las cuales son categorizadas de tres maneras:

\section{- Prácticas racionales administrativas:}

Son las encargadas de organizar y coordinar la estrategia, tales como pronósticos e indicadores, entre otros.

\section{- Prácticas discursivas:}

Hacen referencia a la parte lingüística, cognitiva y simbólica; en estas, existen dos tipos de prácticas que se interrelacionan entre sí: los discursos de estrategia, y las herramientas y técnicas que proporcionan el lenguaje para estos.

\section{- Prácticas episódicas:}

Son los espacios de interacción de los practicantes, tales como talleres, reuniones, etc.

\subsection{Estrategia ambiental}

A partir de la Cumbre de la Tierra de Río de Janeiro llevada a cabo en 1992, la preocupación por los temas ambientales y las presiones que estos suponen para las organizaciones, marcan un punto de ruptura en la forma de hacer negocios, y especialmente, en la forma en que los líderes empresariales conciben a la organización (Esty \& Winston, 2006; Roome, 1992). Lo anterior, dio origen al concepto de sostenibilidad empresarial definida como "la satisfacción de las necesidades de los stakeholders directos e indirectos [...] sin comprometer la capacidad de satisfacer las necesidades de los stakeholders en el futuro" (Dyllick \& Hockerts, 2002) [Traducción de los autores].

Las primeras aproximaciones al estudio de la gestión ambiental en las organizaciones, se centraron en identificar las ventajas en la rentabilidad empresarial (Porter \& Van der Linde, 1995; Aragón-Correa et al., 2005). Debido a su carácter estratégico, el Enfoque Basado en Recursos (EBR) fue acogido rápidamente, dando origen al Enfoque Basado en Recursos Naturales (EBRN) propuesto por Hart (1995).

Este enfoque, en el que los aspectos ambientales son también estratégicos para la organización, llevó a pensar en el concepto de Estrategia Ambiental, concebida como un "conjunto de iniciativas que mitigan el impacto de una organización sobre el medio ambiente" (Walls \& Phan, 2011, p.73) [Traducción de los autores]. La estrategia ambiental de la organización se materializa en la selección de una serie de prácticas a poner en marcha en la empresa y en el grado en el que las mismas se desarrollan y son coherentes entre sí (Aragón-Correa et al., 2005).

Para Roome (1992), existen tres corrientes entre las organizaciones: corriente non-compliance, en donde las organizaciones no responden de ninguna forma a las presiones sociales o legales -normativas- sobre aspectos ambientales, bien por desinterés o por falta de recursos y capacidades que les permitan afrontar estos retos; corriente compliance, en donde las organizaciones reconocen problemas ambientales puntuales y responden a ellos de acuerdo con la normatividad -generalmente con medidas de final del tubo-. Estas organizaciones no anticipan ninguna medida que permita mejorar su desempeño ambiental en el futuro. Finalmente, corriente compliance plus, en donde las organizaciones conciben la estrategia ambiental como un aspecto fundamental del negocio, incluyen estrategias y políticas que alinean los objetivos de la organización con el desempeño ambiental, el cumplimiento de la norma y la satisfacción de las expectativas en términos ambientales de los Stakeholders de la organización (Esty \& Winston, 2006; Rodriguez, Magnan \& Boulianne, 2013). Algunos autores coinciden en referirse a las estrategias ambientales como reactivas y proactivas, para representar las corrientes organizacionales anteriormente mencionadas (Walls \& Phan, 2011; Buysse y Verbecke, 2003; Moreno-Mantilla et al., 2013; Aragón-Correa et al., 2005). 
Buysse y Verbeke (2003), indican que la configuración de estrategias ambientales proactivas requiere de esfuerzos simultáneos de parte de la organización en los siguientes ámbitos: reverdecimiento de productos y procesos de manufactura; capacitación de los empleados y participación de los mismos en la solución de problemáticas ambientales; involucramiento de las áreas funcionales en la gestión ambiental de la organización; implementación de sistemas y herramientas de gestión ambiental, incluida alguna forma de Análisis del Ciclo de Vida; y finalmente, declaración explícita de mejoramiento del desempeño ambiental en la planeación estratégica de la compañía.

\subsection{La estrategia ambiental en Pequeñas y Medianas Empresas (PyMEs)}

El desarrollo teórico sobre estrategia ambiental, así como su validación empírica, han sido enfocados tradicionalmente en grandes organizaciones. Los trabajos existentes en gestión ambiental han prestado "muy escasa atención a las PyMEs, (...), este desequilibrio resulta especialmente inapropiado si consideramos que las PyMES constituyen cerca del $90 \%$ de todas las organizaciones existentes (...), y se estima que generan más del 50\% de la contaminación mundial" (Hillary, 2000. Citado por Aragón-Correa et al., 2005).

La literatura sobre estrategia ambiental, generalmente ha mostrado que diversas limitaciones de tipo financiero, tecnológico u organizacional, hacen menos probables las iniciativas voluntarias de carácter estratégico, para ir más allá de la norma, en el caso de las Pequeñas y Medianas Empresas (Tilley, 1999; Mir \& Feitelson, 2007). Bajo la perspectiva del EBR, son tres las capacidades organizacionales que definen el éxito de las estrategias ambientales en las PyMEs: la capacidad de gestionar los intereses de los Stakeholders; la proactividad estratégica; y el liderazgo compartido, con participación de todas las áreas y miembros de la organización (Aragón-Correa et al., 2005).

No obstante, innovaciones estratégicas en términos de sostenibilidad también son posibles en este tipo de empresas, como lo han revisado recientemente Klewitz y Hansen (2014). Estos autores proponen un marco de referencia que, de acuerdo con el grado de integración

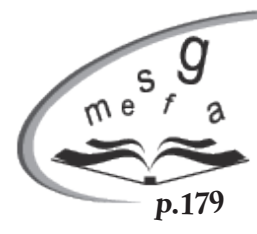
de los aspectos sociales/ambientales a productos, procesos y estructuras organizacionales, clasifica el comportamiento de las PyMEs como resistente, reactivo, anticipatorio, basado en innovación o cimentado en sostenibilidad.

Existe evidencia empírica sobre la existencia de tres grupos de PyMEs en Colombia, en cuanto a su comportamiento ambiental estratégico. Un primer grupo de líderes, en donde las organizaciones incluyen estrategias proactivas relacionadas con la implementación de alguna forma de Análisis del Ciclo de Vida (Moreno-Mantilla et al., 2013); un segundo grupo de empresas "seguidoras» de las del primer grupo, las cuales implementan algunas prácticas de eco-eficiencia con énfasis en los procesos de producción, en línea con lo que Esty y Simmons (2011) definen como jugadas intermedias. Y finalmente, existe un tercer grupo de empresas reactivas, o cumplidores a la fuerza, que no evidencian contar con una visión estratégica de lo ambiental (Moreno-Mantilla \& Reyes, 2010). 


\section{METODOLOGÍA}

\begin{abstract}
partir del marco teórico integrado por los aportes fundacionales de Mintzberg (1985, 1987), Jarzabkowski (2005) y, Jarzabkowski y Spee $(2007)^{2}$, los autores se proponen identificar cómo las PyMEs configuran su estrategia ambiental, Para ello, se desarrolló un estudio de carácter descriptivo, siguiendo una estrategia investigativa (Creswell, 2009) de Estudio de Caso Múltiple (Yin, 2003). El estudio conlleva a seleccionar casos representativos independientes y a diseñar protocolos de recolección y análisis de información aplicable a cada uno de los casos.
\end{abstract}

Los casos fueron elegidos de acuerdo con el alcance de la investigación: empresas participantes en el segundo nivel (Producción sostenible) del PGAE de la SDA. Con el apoyo de la SDA se seleccionaron dos empresas, una de producción y una de servicios, que resaltaron por su interés en avanzar hacia la implementación de prácticas ambientales proactivas. Dicha medida de proactividad fue evaluada de acuerdo consu desempeño en la etapa ACERCAR, es decir, primer nivel del PGAE. El interés en estudiar una empresa de producción y una de servicios, obedece a la necesidad de encontrar patrones que permitan generalizar los resultados obtenidos, independientemente de la naturaleza de las actividades de la organización.

Los investigadores participaron activamente en la formulación de un plan estratégico ambiental en las empresas estudiadas, por medio de un programa de diez sesiones a lo largo de cuatro meses aproximadamente, conformado de la siguiente manera:

- Sesión I: contextualización del programa de formulación de un Plan Estratégico Ambiental.

- Sesión II: Desarrollo de Matriz de Materialidad, Energía y Desechos (MED) (Van Hoof, Monroy y Saer, 2008).
- Sesión III: análisis de resultados obtenidos en Matriz MED.

- Sesión IV: desarrollo de la herramienta Aspects, Upstream, Downstream, Issues, Opportunities (AUDIO) (Esty \& Winston, 2006).

- Sesión V: análisis de resultados obtenidos en AUDIO. Sesión VI: diagnóstico de Materialidad Interna (Rodriguez \& Ricart, 1998).

- Sesión VII: diagnóstico de Materialidad Externa (Aragón-Correa et al., 2005).

- Sesión VIII: desarrollo Matriz de Materialidad (Esty \& Simmons, 2011).

- Sesión IX-X: formulación del Plan Estratégico Ambiental.

La participación en este programa, dio la posibilidad a los autores de usar las siguientes fuentes de información:

- Observación participativa: permitió realizar observaciones directas sobre los tomadores de decisión al interior de la organización, las dinámicas internas de la misma y las interacciones con su exterior.

- Instrumentos: adicionalmente al trabajo de observación participativa, el acompañamiento de los autores permitió el uso de las herramientas descritas en el programa: Matriz MED, Matriz AUDIO, diagnóstico de Materialidad Interna, Diagnóstico de Materialidad Externa y Matriz de Materialidad.

- Entrevistas: fueron realizadas a los miembros de la organización directamente relacionados al PGAE de la SDA. Se indagó el interés de la organización y de sus directivas por ejecutar iniciativas ambientales la visión estratégica ambiental al interior de las organizaciones, y los aspectos cuya información proveniente de las otras fuentes no se consideró suficiente.

\footnotetext{
2 Se dirige al lector a trabajos recientes de Jarzabkowski (Jarzabkowski, Bednarek y Cabantous, 2014; Jarzabkowski y Kaplan, 2014; Balogun et al., 2014; Smets et al., 2014), en donde se aborda en profundidad la aplicación de una estrategia de investigación etnográfica en el campo de estudios sobre Strategy as Practice, frente a la estrategia de estudio de caso con que los autores del presente trabajo han abordado el estudio.
} 
Los datos recolectados por los investigadores se analizaron mediante la técnica de Cross-case. Yin (2003), manifiesta que esta técnica es relevante en estudios de caso múltiple, ya que permite la construcción de conclusiones robustas. El uso de Word Tables permitió organizar la información de cada caso individual bajo ciertos parámetros acordes con el estudio, facilitando el análisis de la información y la obtención de conclusiones.
La validez del estudio está soportada por los criterios sugeridos por Yin (2003): validez de constructo, debido al uso de múltiples fuentes de evidencia; validez externa, debido a la replicación del estudio en las unidades de análisis; y fiabilidad, debido al uso del protocolo para la recolección de datos. Dado el carácter descriptivo del estudio no aplica la evaluación de la validez interna.

\section{RESULTADOS}

\subsection{Caso A}

La organización objeto de estudio se dedica a la producción y comercialización de productos alimenticios, cuenta con más de 20 años en el mercado nacional y su marca es una de las más reconocidas en su segmento. A partir de 2005, la empresa efectúa alianzas comerciales con grandes superficies, parques temáticos y almacenes especializados, los cuales se convierten en socios estratégicos de la compañía. Es precisamente por intermediación de uno de ellos, que se inician los esfuerzos ambientales de la organización al ser invitados a hacer parte del PGAE de la SDA.

Las primeras observaciones permitieron identificar la voluntad de la organización en avanzar hacia un modelo de producción ambientalmente sostenible, un claro interés en ir más allá del cumplimiento normativo y la necesidad de mejorar su desempeño ambiental. Además, la organización identifica en ello una posible fuente de ventaja competitiva, representada principalmente, según lo expresado por su gerencia administrativa y de operaciones, en que "es importante para los clientes saber [sic] que se está haciendo algo por el medio ambiente".

Los primeros esfuerzos ambientales de la organización provienen de la visión de calidad integral. Paralelamente al PGAE y al programa de formulación de un plan estratégico ambiental, la compañía está involucrada en la certificación de sus procesos en ISO 9000, ISO 14000 e ISO 18000. Según lo expresado por el administrador del sistema de gestión de la empresa, la normatividad rigurosa y las dinámicas del mercado, generan su interés por obtener certificaciones que permitan mejorar sus procesos. Sin embargo, también hace precisión en que las iniciativas ambientales anteriores a su vinculación en el PGAE y al acompañamiento de los autores, constituían pequeños avances: básicamente, clasificación de residuos ordinarios al interior de la empresa y en los puntos de venta, por lo que el gran avance en estrategia ambiental se da tras la vinculación al programa de la SDA.

En la actualidad, la empresa está desarrollando métricas que le permiten dar seguimiento a sus problemáticas. De igual manera, ha implementado los primeros programas de control de residuos, trampas de control de grasa a vertimientos; al interior de la organización se propone la creación del departamento de gestión ambiental. Este comportamiento se ajusta al de una estrategia impuesta. Cabe resaltar que las actividades ambientales ejecutadas hasta el momento en la organización, no hacen parte aún de una formulación estratégica ambiental declarada.

Frente a estas primeras muestras de proactividad ambiental, se pudo identificar que la responsabilidad de la formulación y planeación de las iniciativas ambientales, recae en la gerencia media de la organización, concretamente en el administrador del sistema de gestión y eventualmente en el gerente administrativo y de operaciones. Estos, bajo la perspectiva de SAP, se comportan como practicantes. 
Mediante una entrevista realizada al administrador del sistema de gestión, se determinó que la alta gerencia apoya todas las iniciativas ambientales ejecutadas en la organización, principalmente aprobando los presupuestos requeridos para las misma y la participación de la empresa en los diversos programas ambientales anteriormente descritos.

Por otra parte, la definición de practicante permite incluir agentes externos a la organización. En este caso, es clara la influencia tanto del regulador, representado por la SDA, como del socio empresarial que los acompaña e invita a hacer parte del PGAE, y de la academia, representada por un profesor y dos estudiantes tesistas de pregrado, quienes se han encargado de apoyar a la empresa en el proceso. Así mismo, las actividades que se ejecutaron dentro del programa de formulación estratégica ambiental, podrían ser vistas como prácticas, ajustándose a los tres tipos descritos por Jarzabkowski: prácticas administrativas, las cuales son las herramientas de diagnóstico y formulación brindadas por los autores a las empresas; prácticas episódicas, representadas por los encuentros que sostiene la empresa con la SDA, las reuniones que tuvieron con los autores, y en general, por los encuentros dispuestos al interior de la organización para poder desarrollar su estrategia ambiental; finalmente, las prácticas discursivas, que corresponden al contexto teórico y discursivo que permite abordar en un mismo lenguaje los asuntos ambientales.

Finalmente, las praxis que se derivan del plan estratégico ambiental no pudieron ser identificadas debido al alcance del estudio, el cual llega hasta la formulación del plan y no hasta su implementación. Sin embargo, se reconoce una praxis anterior a la formulación del PEA, evidenciada en la separación en la fuente y posterior venta de residuos orgánicos.

\subsection{Caso B}

La segunda organización objeto de estudio, es una mediana empresa de servicios del sector automotor fundada en Colombia hace seis décadas. Esta cuenta con varias sucursales, siendo la sucursal estudiada la líder en la implementación de nuevos programas en la compañía. Los esfuerzos ambientales inician después de haber ganado licitaciones con algunas entidades públicas, lo que permite formalizar la relación con la SDA, quien lo invitó a participar en el PGAE. Antes de esta invitación, el desempeño ambiental de la organización se limitaba al cumplimiento de la legislación, referido a la disposición controlada de residuos peligrosos.

Aunque la compañía ha realizado iniciativas ambientales como el aprovechamiento de la luz natural, la separación en la fuente de los residuos, la racionalización de la energía eléctrica y del recurso hídrico, es la primera vez que emprende la formulación de un Plan Estratégico Ambiental. La alta gerencia, ha empezado a identificar la importancia de un desempeño ambiental más allá de la norma. Sin embargo, para conseguirlo fue necesario, según lo expresado por el jefe de taller, "romper mitos y vender la idea a la gerencia", aunque "en este momento estamos en una fase de concientización (sic) a la gerencia [media] y alta gerencia".

La organización, durante su proceso de formulación, dio evidencia de la existencia de tres tipos de estrategias deliberadas. Por un lado, se tiene la estrategia planeada, en donde los estrategas que participaron es decir, la gerencia media, están en proceso de articular detalladamente un documento con las futuras acciones de la organización. Por otro lado, está la estrategia emprendedora, en donde la sucursal estudiada espera el éxito en la imple-mentación de las estrategias para replicarlas en las demás sucursales. Finalmente, está la estrategia impuesta, debido a que fue iniciativa de la SDA, uno de sus entes reguladores, incluir a la organización en el ejercicio de formulación de un plan estratégico ambiental.

Se identificaron dos practicantes internos como los principales configuradores de la estrategia ambiental. Su rol de liderazgo se debió a la participación directa en el programa de la SDA. Por un lado, se tiene al jefe de taller, que cuenta con 16 años de experiencia en las actividades operativas de la organización, y por el otro, la coordinadora ambiental, una practicante que cuenta con conocimientos técnicos y una nueva perspectiva de la compañía. A su vez, existen practicantes internos 
pasivos, vinculados al desarrollo de unas pocas prácticas que contribuyeron a la estructuración de la estrategia.

En cuanto a los practicantes externos, los actores más representativos son la SDA y la academia. Los talleres o reuniones realizados en el proceso, fueron identificados como prácticas episódicas; estos escenarios de interacción, en la mayoría de los casos, fueron organizados por los académicos y realizados en las instalaciones de la organización. No obstante, la empresa también generó espacios para realizar talleres en compañía de los practicantes internos pasivos y bajo la coordinación de los practicantes internos activos.
También, se identificaron prácticas administrativas a través del uso de herramientas, de instructivos y asesorías ofrecidas por los autores, quienes fueron los encargados de transmitir a los practicantes internos el vocabulario sobre estos temas provenientes de la literatura conocidas como prácticas discursivas-.

Se identificaron praxis durante la formulación del plan, como es el reemplazo de vasos desechables por vasos de porcelana en las actividades diarias de la organización, buscando así reducir la generación de desechos sólidos (900 vasos desechables por mes), sin embargo, se desconoce el cambio en un índice de impacto ambiental medido a través de una metodología de Análisis de Ciclo de Vida.

\section{DISCUSIÓN DE RESULTADOS}

$\mathbf{L}$ as dos organizaciones iniciaron su participación en el PGAE en 2013, debido a la presión ejercida por los clientes (caso B) o socios empresariales (caso A), que pueden verse como algunos de los Stakeholders con mayor influencia sobre las organizaciones para llevarlas a formular un plan o estrategia ambiental (Henriques \& Sadorsky, 1996). Durante el primer nivel del programa, las organizaciones realizaron los primeros acercamientos a los temas ambientales. Antes de la participación en el PGEA, las se habían enfocado únicamente en cumplir la normatividad ambiental asociada a su actividad, desarrollando en ambos casos estrategias como una respuesta al ente regulador. Las iniciativas ambientales realizadas no estaban ligadas a los aspectos de competitividad de la organización, y por lo tanto, no generaban mayor impacto favorable sobre el desempeño del negocio.

Debido a que la estrategia se relaciona directamente con la organización (Whittington, 2003), los practicantes son pieza fundamental en la configuración de estrategias ambientales. Estos pueden contar con objetivos distintos entre sí, aunque con una meta en común relacionada a la proactividad ambiental. Cada practicante, sea individuo o grupo, contribuye en la configuración de la estrategia dependiendo de su objetivo, habilidad, conocimiento y potestad sobre la organización. El estudio de caso múltiple, permitió identificar como practicantes principales para el Caso A, al administrador de calidad y al ingeniero Junior; y como practicantes pasivos, al gerente de planta, al asesor de seguridad industrial y salud ocupacional, y al gerente de administración y operaciones. Para el Caso $\mathrm{B}$, los practicantes principales fueron, el jefe de taller y la coordinadora ambiental, y los practicantes pasivos, el gerente financiero, gerente del talento humano, jefe de operaciones comerciales y coordinador de calidad.

Se encontró que son pocos los practicantes internos directos y que estos, por lo general, pertenecen a la gerencia media y operacional. Esto, pese a que el programa de formulación de un plan estratégico ambiental, diseñado por los autores, concebía la participación de la alta y media gerencia, así como del equipo técnico relacionado con procesos y actividades internas en la organización. La participación en las actividades del programa, se vio reducida a los ya 
mencionados miembros de la organización. Lo anterior indica la dificultad de integrar, tanto vertical como horizontalmente, la visión estratégica ambiental y evidencia una inconsistencia con lo planteado en la NTC-ISO 14001 (2004), donde se asegura, que el éxito de los sistemas de gestión depende especialmente de la participación de la alta gerencia.

La participación de esta última en ambas organizaciones, no fue observada de forma directa por los investigadores. De otra parte, se pudo constatar la intención de la gerencia media en la construcción de estrategias ambientales, como lo es la participación en el programa de la SDA, por lo que estas se consideran deliberadas para las dos empresas. Sin embargo, no es conveniente ignorar la existencia de estrategias ambientales emergentes que puedan surgir en el futuro, originadas a partir de los esfuerzos deliberados que actualmente se están desplegando.

Es importante resaltar que en el enfoque de SAP, los participantes externos observados como grupos, cumplen papeles fundamentales en la contribución de la configuración de la estrategia, tal como acompañamiento, apoyo, control, tutoría y demanda, entre otros. Uno de los practicantes externos más significativos en el caso bajo análisis, es la SDA, quien ha estado cumpliendo la función de acompañamiento para las empresas. Aunque desde la perspectiva de las compañias actúa fundamentalmente como un ente de control, la literatura da muestras de un rol cambiante del regulador, avanzando a un modelo de interacción y cooperación con los Stakeholders, como lo refiere Moreno-Mantilla (2007).

De igual manera, se encontró que la academia se ha encargado de apoyar a las empresas durante este proceso. Es importante aclarar que la participación de la academia se da, en este caso, a través de una alianza informal con el regulador. Bajo la recomendación de la SDA, las empresas le abrieron sus puertas para permitirle ser parte activa del proceso. De esta manera, sería erróneo considerar a la academia como un practicante externo, individual e independiente de la SDA.
Los distintos individuos y grupos se relacionan entre ellos a través de interacciones, ya sea en escenarios generados por acuerdo mutuo, o por la iniciativa de tan solo uno de ellos. De esta manera, los participantes interactuaron a través de la creación de espacios es decir, prácticas episódicas como talleres y reuniones, organizados por los autores y la SDA. De su parte, las organizaciones también se encargaron de crear espacios temporales, no sistemáticamente, que permitieron la participación de los practicantes pasivos en el uso de herramientas de diagnóstico y en un proceso consultivo desarrollado en el marco de la formulación del PEA.

Las herramientas de diagnóstico, como prácticas administrativas, aportaron información representativa para la construcción de estrategias ambientales. La capacitación en el uso de estas fue realizada por los autores, quienes establecieron los puntos de partida, condiciones y terminología a utilizar llamadas prácticas discursivas. Las empresas mostraron diferencias en el grado de uso y apropiación de estas últimas, debido presumiblemente a los antecedentes académicos de los practicantes principales.

En ambos casos, se observó que las iniciativas ambientales nacen no de la alta gerencia sino de la gerencia media, quien se encarga de diseñar y difundir las acciones que se llevarán a cabo en la organización, luego de contar con el aval de la alta gerencia. La visión estratégica de los temas ambientales, recae en un grupo reducido de miembros y son estos los que intentan transmitirla al resto de la compañía mediante la formulación de un plan. El comportamiento con-cuerda con el de una estrategia planeada.

Sin embargo, debido a la presión que ejerce la SDA sobre la organización, dichas iniciativas también podrían ser consideradas como una estrategia impuesta. Este tipo de estrategia es común en las pequeñas y medianas empresas debido a su relación con el regulador. Ellas cumplen actualmente con la normatividad ambiental, pero además demuestran la intención de avanzar hacia un modelo que incluya los aspectos ambientales como parte estratégica de la organización, lo que podría ser identificado como evidencia de proactividad ambiental. 
Figura 2. Síntesis de los hallazgos del estudio de caso

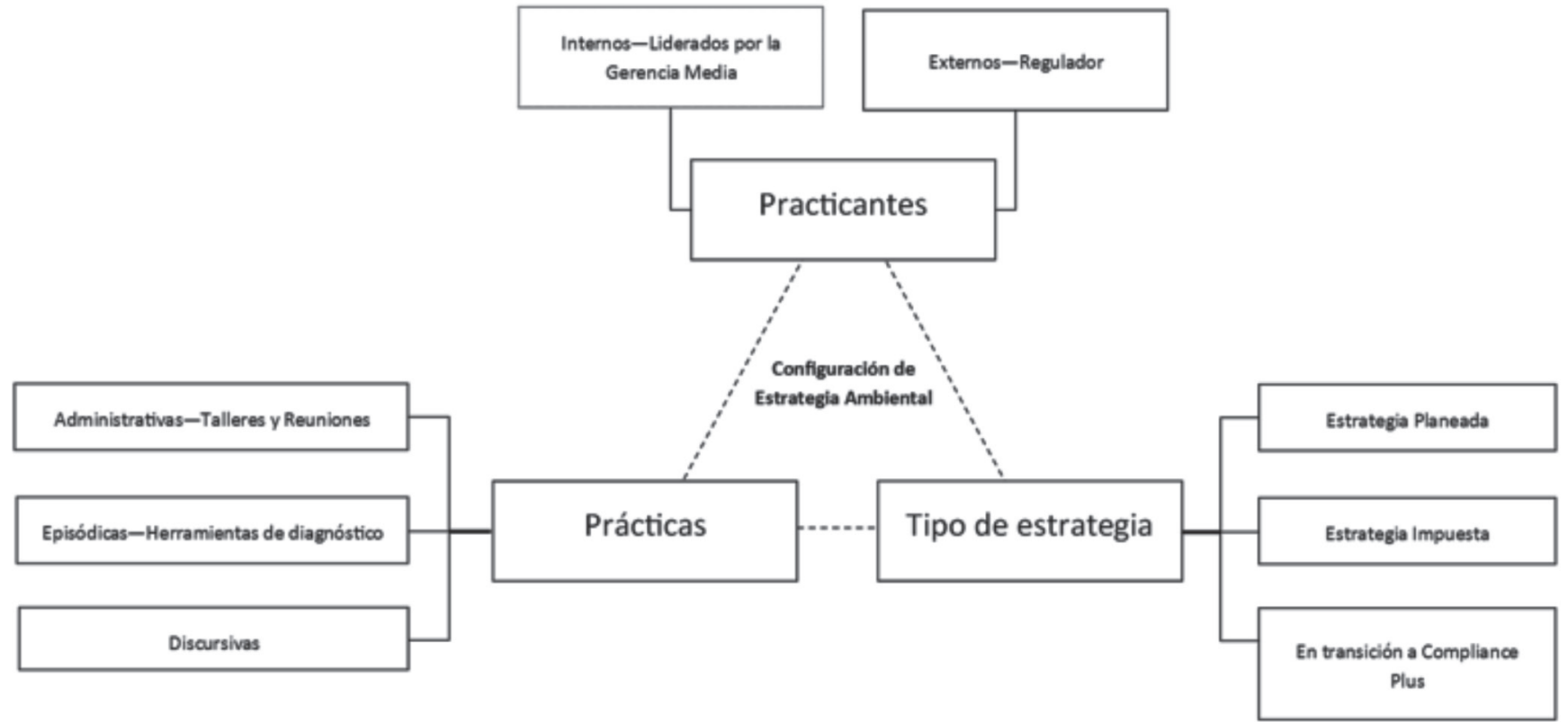

Fuente. Elaboración propia de los autores, (s.f).

Tabla 1. Síntesis de los hallazgos del estudio de caso

\begin{tabular}{|c|l|}
\hline \multicolumn{2}{|c|}{ Configuración de una estrategia ambiental } \\
\hline \multirow{4}{*}{ Practicantes } & $\begin{array}{l}\text { Participación de practicantes internos activos (cargos de gerencia media y } \\
\text { operacional), y en menor medida de la alta gerencia. } \\
\text { Participación de la SDA - principal stakeholder-y la academia como practicantes } \\
\text { externos, cumpliendo papeles de apoyo y auditoría. } \\
\text { Limitada inclusión de practicantes pasivos. }\end{array}$ \\
\hline \multirow{5}{*}{ Prácticas } & $\begin{array}{l}\text { Prácticas administrativas: uso de herramientas de diagnóstico. } \\
\text { Prácticas discursivas: capacitaciones recibidas sobre el uso y terminología de las } \\
\text { herramientas de diagnóstico. } \\
\text { Prácticas episódicas: creación de espacios para la realización de talleres y reuniones. }\end{array}$ \\
\hline \multirow{5}{*}{ Tipo de estrategias } & $\begin{array}{l}\text { Principalmente de tipo deliberada, lo que se infiere de la intención de las gerencias } \\
\text { medias de participar en los programas ambientales de la Secretaría Distrital. }\end{array}$ \\
\cline { 2 - 3 } & $\begin{array}{l}\text { De tipo planeada, en donde los practicantes internos son los responsables de } \\
\text { articular y difundir el PEA. }\end{array}$ \\
\cline { 2 - 3 } & De tipo impuesta, debido a la presión ejercida por el regulador. \\
\cline { 2 - 3 } & $\begin{array}{l}\text { En transición a la corriente compliance plus, partir de unos primeros indicios de } \\
\text { proactividad ambiental. }\end{array}$ \\
\hline
\end{tabular}

Fuente. Elaboración propia de los autores, (s.f). 


\section{CONCLUSIONES}

$\mathbf{L}$ a ejecución del estudio de caso bajo marcos teóricos disímiles, es decir estrategia deliberada y emergente, frente a la perspectiva SAP, permitió analizar de manera comprensiva el comportamiento de las compañías, obteniendo una descripción más profusa y compleja. Más allá de facilitar una taxonomía de la estrategia ambiental, confirmó la necesidad de dar importancia a los actores humanos como los gestores de estrategia en las organizaciones, tal y como lo sugiere SAP (Whittington, 2003). Estos gestores internos, reciben una atención especial en enfoques organizacionales tales como la Teoría de Stakeholders o la Visión de la Empresa Basada en Recursos. En el primer caso, los requerimientos y expectativas (Aschehoug et al., 2012) frente al desempeño ambiental de la organización pueden provenir de actores internos como los dueños, la gerencia o los empleados en una organización (Henriques \& Sadorsky, 1996). En el segundo caso, Hart (1995) ha propuesto que la prevención de la contaminación depende de la disponibilidad y el desarrollo de un recurso tácito que está embebido en las personas, como lo es el capital humano. Similarmente, la implementación de una estrategia de tutelaje de producto (Hart, 1995) depende de la coordinación interfuncional de diferentes cargos y funciones que recaen en los actores humanos al interior de una organización.

El estudio de caso permitió observar que con anterioridad a la participación de las organizaciones en el PGAE de la SDA, la estrategia ambiental se orientó al cumplimiento de la legislación. Después de haber iniciado el programa de formulación de un plan estratégico ambiental, las estrategias se consolidan como estrategias deliberadas de tipo planeada, y siguen siendo resultado de la presión de agentes externos como el regulador y clientes o socios empresariales, es decir, de tipo impuesta. Por consiguiente, puede afirmarse que las empresas estudiadas están realizando la transición de una estrategia Compliance a una del tipo Compliance Plus, ubicándose en el grupo de las PyMES seguidoras.
El estudio ofrece evidencia de que la gerencia media se constituye como el practicante principal al interior de estas PyMEs, y que en ella, recae la responsabilidad de diseñar y ejecutar las iniciativas ambientales. Los practicantes externos cumplen las funciones de apoyo, control y tutoría, actuando como precursores de los esfuerzos ambientales en las organizaciones. El ente regulador interviene como el practicante externo principal.

La configuración de la estrategia ambiental requiere de un proceso de formulación, compuesto de herramientas de diagnóstico y escenarios de interacción, con la mediación de prácticas discursivas. Estos escenarios facilitan el involucramiento de todos los actores.

La participación de las empresas en el PGAE, junto con la formulación de un Plan Estratégico Ambiental, permitió la participación de los empleados en la solución de problemáticas ambientales y la implementación de herramientas de gestión ambiental. Lo anterior, es un indicio de la existencia de proactividad ambiental en la empresa.

Las empresas demostraron capacidad para emprender el camino de aprendizaje organizacional que permitió reconocer a sus partes interesadas, así como sus demandas y expectativas frente a los aspectos ambientales de la organización. Lo anterior, sumado a su proactividad ambiental al plantear estrategias que respondan a esas demandas y expectativas, señala la posibilidad de éxito de las estrategias ambientales, representado en el nivel de satisfacción de dichas expectativas y en el impacto positivo sobre la competitividad del negocio.

Las condiciones encontradas no permitieron observar ninguna estrategia implementada, debido a que el alcance del estudio contempló hasta la formulación de las estrategias ambientales, lo cual constituye una limitación de este trabajo. Un seguimiento posterior que evalué las estrategias en funcionamiento, permitirá profundizar en la comprensión de las praxis, que es uno de los conceptos esenciales al enfoque SAP. 


\section{REFERENCIAS BIBLIOGRÁFICAS}

Aragón-Correa, J. A., García-Morales, V. J., \& Hurtado-Torres, N. E. (2005). Un modelo explicativo de las estrategias medioambientales avanzadas para pequeñas y medianas empresas y su influencia en los resultados. Cuadernos de Economía y Dirección de la Empresa, 25, pp. 29-52.

Aschehoug, S. H., Boks, C., \& Storen, S. (2012). Environmental information from stakeholders support-ing product development. Journal of Cleaner Production, 31, pp. 1-13.

Buysse, K., Verbeke, A. (2003). Proactive environmental strategies: a stakeholder management perspective. Strategic Managemnt Journal, 24(5), pp 453-470.

Carter, C., Clegg, S. R., \& Kornberger, M. (2008). Strategy as practice? Strategic Organization, 6, 83-99.

Çetinkaya, Ö., \& Kalkan, A. (2013). Strategy Focus in Turkish SMEs: Emergent or Deliberate Strate-gies? Science Direct, 99, pp. 83-99.

Creswell, J. (2008). Research Design: Qualitative, Quantitative, and Mixed Methods Approaches. Estados Unidos: SAGE Publications, Inc.

De Bakker, F., \& Nijhof, A. (2002). Responsible chain management: a capability assessment frame-work. Business Strategy and the Environment, 11(1), pp 63-75.

Dyllick, T., \& Hockerts, K. (2012). Beyond the business case for corporate sustainability. Business Strategy and the Environment, 11(1), pp. 130-141.

Esty, D. C., \& Winston, A. S. (2006). Green to Gold: How Smart Companies Use Environmental Strategy to Innovate, Create Value, and Build Competitive Advantage. New Haven and London: Yale Uni-versity Press.

Esty, D. C., \& Simmons, P. J. (2011). The green to Gold Business Playbook: How to Implement Sustainability Practices for Bottom-Line Results in Every Business Function. Hoboken, New Jersey: John Wiley \& Sons, Inc.

Fuller-Love, N., \& Cooper, J. (2000). Deliberate versus emergent strategies: a case study of infor-mation technology in the Post Office. International Journal of Information Management, 20, pp. 209-223.

Hart, S. L.,(1995). A Natural-Resource-Based View of the Firm. The Academy of Management Review, 20(4), pp. 986-1014.

Helfat, C.E., Finkelstein, S., Mitchelll, W., Peteraf, M.A., Singh, H., Teece, D.J., \& Winter, S.G. (2007) Dynamic Capabilities: Understanding Strategic Change in Organizations. Singapore: Blackwell Publishing.

Henriques, I., \& Sadorsky, P. (1996) The determinants of an environmentally responsive firm: An empirical approach. Journal of Environmental Economics and Management, 30(3), pp. 381-395. 
Instituto Colombiano de Normas Técnicas y Certificación (2004). Sistemas de gestión ambiental. Requisitos con orientación para su uso. NTC-ISO 14001. (s.c).

Jarzabkowski, P. (2005). Strategy as Practice: An Activity-Based Approach. Londres: SAGE Publican-tions Ltd.

Jarzabkowski, P., Bednarek, R., \& Cabantous, L. (2014). Conducting global team-based ethnography: Methodological challenges and practical methods. Human Relations.

Jarzabkowski, P., \& Kaplan, S. (2014). Using strategy tools in practice: An exploration of technologies of rationality in use. Strategic Management Journal.

Jarzabkowski, P., Mantere, S., \& Vaara, E. (2014). Placing Strategy Discourse in Context: Socio-materiality, Sensemaking and Power. Journal of Management Studies, 51(2), pp. 275-291.

Jarzabkowski, P., \& Spee, A. P. (2009). Strategy-as-practice: A Review and Future Directions of the Field. International Journal of Management Reviews, 11(1), pp. 69-95.

Klewitz, J., \& Hansen, E. G. (2014). Sustainability-oriented innovation of SMEs: a systematic review. Journal of Cleaner Production, 65, 57-75.

Mintzberg, H., \& Waters, J. (1985). Of strategies, Deliberate and Emergent. Strategic Management Journal, 6(3), 257-272.

Mintzberg, H. (1987). The Strategy Concept I: Five Ps for Strategy. California Management Review, Julio-Agosto, pp. 11-24.

Mir, D. F., \& Feitelson, E. (2007). Factors affecting environmental behaviour in micro-enterprises laun-dry and motor vehicle repair firms in Jerusalem. International Small Business Journal, 25(4), pp. 383-415

Moreno-Mantilla, C. E.,(2007) Adopción de tecnologías más limpias en firmas industriales: un estudio multimétodo sobre el efecto de la aplicación de límites de vertimiento y tasas retributivas en Santander, Colombia. Bogotá: Cuadernos de administración, Pontificia Universidad Javeriana.

Moreno-Mantilla, C. E., \& Reyes, J. F. (2010). Environmental strategy and organizational capabilities: An exploration of the Natural-Resource-Based View with a focus on Colombian firms. In: Riihelä T, Mattila M (eds) COMBI 2010 : Contemporary Views on Business: Partnering for the Future., pp. 16-43.

Moreno-Mantilla, M. C., Romero-Larrahondo, P. A., \& Reyes-Rodriguez, J. F. (2013). Driving product stewardship: an empirical evaluation of the association between some form of LCA implementation and environmental strategy choice in Colombian firms. Mendoza: Fifth International Conference on Life Cycle Assessment CILCA2013.

Porter, M. (1996). What is Strategy? Harvard Business Review, Noviembre- Diciembre, pp 61-78.

Porter, M., \& Van der Linde, C. (1995). Green and Competitive: Ending the Stalemate. Harvard Business Review, Septiembre- Octubre, pp 120-134. 
Rodriguez, M., Magnan, M., \& Boulianne, E. (2013). Stakeholders influence on environmental strategy and performance indicators: A managerial perspective. Management Accounting Research, 24, pp 301-316.

Rodriguez, M, A., \& Ricart, J, E. (1998). Dirección Medioambiental de la empresa. Gestión Estrátegica del Reto Medioambiental: conceptos, ideas y herramientas. Barcelona: Gestión 2000.

Roome, N. (1992). Developing Environmental Management Strategies. Business Strategy and the Environment, $1(1)$, pp 11-24.

Secretaria Distrital de Ambiente (2014). Programa Gestión Ambiental Empresarial. Recuperdado de: http:// ambientebogota.gov.co/es/web/gae/iicio

Smets, M., Burke, G., Jarzabkowski, P., \& Spee, P. (2014). Charting new territory for organizational ethnography: Insights from a team-based video ethnography of reinsurance trading. Journal of Organizational Ethnography. Forthcoming.

Tilley, F. (1999). The gap between the environmental attitudes and the environmental behaviour of small firms. Business Strategy and the Environment, 8(4), pp. 238-248.

Van Hoof, B., Monroy, N., \& Saer, A. (2008). Producción más Limpia. Paradigma de gestión ambiental. Bogotá: Universidad de los Andes y Alfaomega Colombia.

Walls, J. L., \& Phan, P. H. (2011). Measuring Environmental Strategy: Construct Development, Reliability and Validity. Business \& Society, 50(1), pp 71-115.

Whittington, R. (2003). The work of strategizing and organizing: for a practice perspective. Strategic Organization, 1, pp. 117-125.

Yin, R.K. (2003). Case Study Research: Design and Methods. Estados Unidos: SAGE. 